\title{
Deutscher Forschungspreis geht erstmals nach Österreich
}

Der Preis der Deutschen Akademie der osteologischen und rheumatologischen Wissenschaften ging an die Forschungsgruppe Vinforce am Krankenhaus Barmherzige Schwestern Wien.

Riesengroß war die Freude der heimischen Forschungsgruppe Vinforce rund um Prim. Prof. Dr. Heinrich Resch, der auf dem Fachkongress „Osteologie 2016“ in Essen eine Auszeichnung entgegen nehmen konnte. Der Preis ist kumulativ, er beachtet die Forschungserfolge der jeweils drei Vorjahre (heuer für 2013-2015) im Feld der osteologisch-rheumatologischen Wissenschaften

„Ich bin überglücklich und stolz auf diese internationale Anerkennung und vor allem auf mein Team aus jungen Forscherinnen und Forschern für ihre exzellente und leidenschaftliche Arbeit. Gemeinsam haben wir diesen Preis nach Österreich geholt", erklärte Resch. Krankenhaus-Geschäftsführer und Vinforce-Vizepräsident Thomas Pavek betont: „Besonders wichtig ist uns die Nähe zum klinischen Alltag durch die Forschungsarbeit erhalten unsere Patientinnen und Patienten Behandlungen am aktuellen Stand der Wissenschaft."

) Mit dem Forschungsgruppenpreis würdigt die DAdorW eine herausragende Kollektivleistung auf dem Gebiet der Rheumatologie und Osteologie

Mit dem Forschungsgruppenpreis würdigt die Deutsche Akademie der osteologischen und rheumatologischen Wissenschaften (DAdorW) eine herausragende Kollektivleistung auf dem Gebiet der Rheumatologie und Osteologie. Vinforce beschäftigt sich seit 2005 mit einem breiten Spektrum osteologischer und rheumatologischer Fra-

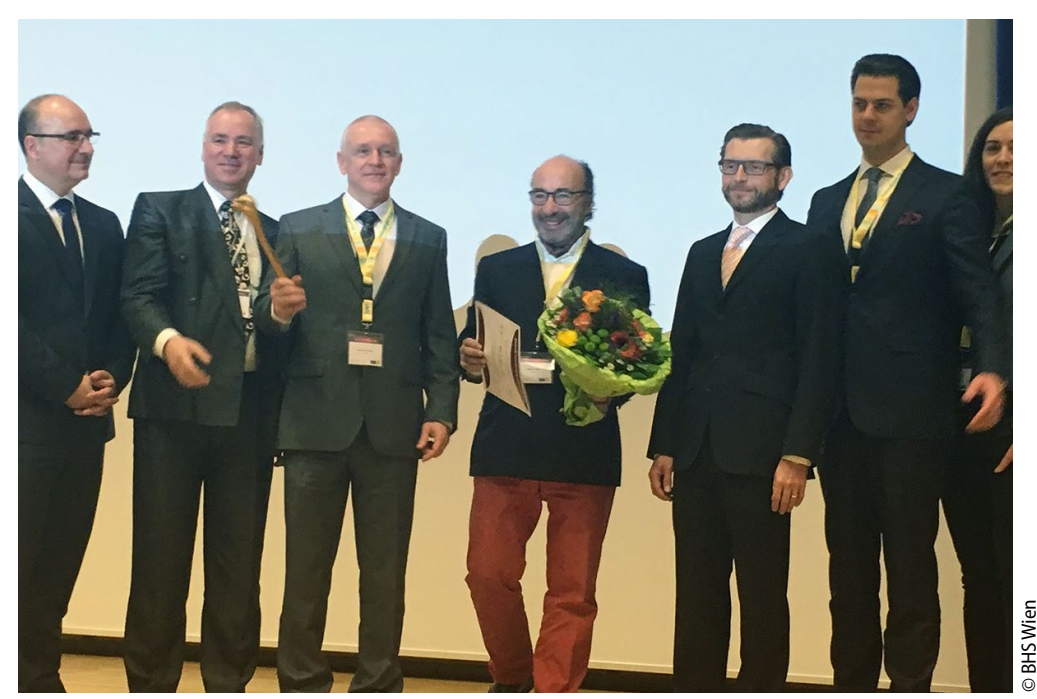

$\Delta$ Prof. Dr. Andreas Kurth, Vorsitzender des DVO Dachverbband der Deutschen Osteologischen Gesellschaften; Dr. Dieter Schöffel, Kongresspräsident Osteologie 2016 Essen; Prof. Dr. Hans Peter Dimai, Präsident der ÖGKM (Österreichische Gesellschaft zur Erforschung des Knochens); Prim. Prof. Dr. Heinrich Resch, Präsident Vinforce und Vorstand II. Med. Abteilung am KH Barmherzige Schwestern Wien; OA Dr. Christian Muschitz, Vinforce und Internist am KH Barmherzige Schwestern Wien; Dr. Roland Kocijan, Vinforce, Gewinner Nachwuchspreis, Internist am KH Barmherzige Schwester Wien; Dr. Judith Haschka, Vinforce, Internistin KH Barmherzige Schwestern Wien

gestellungen von miRNA-Biomarkern über hochauflösende Strukturanalysen bis zu epidemiologischen Untersuchungen.

\section{Friedrich Heuck Osteologie Preis 2016}

Gleichzeitig erhielt Dr. Roland Kocijan, ebenfalls Mitglied von Vinforce und Internist am Krankenhaus Barmherzige Schwestern Wien, den Friedrich Heuck Osteologie Preis 2016 für Erstautoren unter 35 Jahren. Er konnte das internationale Auswahlkomitee mit seiner Arbeit zur Knochenmikroarchitektur bei verschiedenen osteologischen und rheumatologischen Krankheitsbildern besonders beeindrucken. „Der Preis soll zum einen die wissenschaftliche Arbeitsleistung anerkennen, zugleich aber auch Motivation und Ansporn sein, Forschung auf diesem Gebiet fortzusetzen, „so Prof. Dr. Arndt Schilling, Präsident der DAdorW in einer Aussendung.

rheuma plus $2016 \cdot 15: 24$

DOI 10.1007/s12688-016-0070-6

Online publiziert: 31 . Mai 2016

C) Springer-Verlag Wien 2016 